\title{
REJEITOS DE MINERAÇÃO: UM OLHAR DO CENÁRIO BRASILEIRO - PARTE II: TECNOLOGIAS PARA APROVEITAMENTO E NOVOS USOS*
}

\section{Resumo}

Sandra Lúcia de Moraes ${ }^{1}$ Flávia Gutierrez Motta Camila Peres Massola ${ }^{3}$ Eduardo Maziero Saccoccio 4 Marsis Cabral Júnior ${ }^{5}$

Uma característica do setor mineral é a movimentação de grandes volumes de materiais que, em função do teor do bem mineral contido e das particularidades do processo de beneficiamento, ao final gera grandes quantidades de rejeitos. $O$ aproveitamento desses rejeitos é um tema que tem sido discutido e que foi fomentado após o acidente acontecido em 2015, com a barragem da Samarco em Mariana - MG. O trabalho denominado "Rejeitos da Mineração: Um olhar do cenário brasileiro" está estruturado em duas partes. A primeira parte destacou a importância econômica e social do setor mineral para o país. A segunda parte, apresentada neste artigo, aborda o nível de desenvolvimento tecnológico voltado ao aproveitamento dos rejeitos e quais novos usos têm sido propostos. Neste documento, os autores trazem também o fechamento dos levantamentos realizados e suscitam uma discussão visando estimular o desenvolvimento sustentável da mineração, por meio da criação de um novo negócio inserido na cadeia produtiva da mineração.

Palavras-chave: Mineração; Cadeia Produtiva; Rejeitos; Tecnologia.

\section{MINING TAILINGS: A PERSPECTIVE OF THE BRAZILIAN SCENARIO PART II: TECHNOLOGICAL DEVELOPMENT AND NEW USES}

\section{Abstract}

A remarkable characteristic of the mineral activity is the handling of an enormous amount of material, which generates a considerable quantity of tailings, depending on the content of the mineral and the mineral processing technology adopted. Mineral tailings reclamation has become widely discussed, especially after the Samarco's dam spill in 2015. Our work "Mining tailings: a perspective of the Brazilian scenario" is structured in two parts. The first part highlighted the economic and social relevance of the mining activity to the country. The second one, presented in this work, approaches the level of the technological development available for the recovery of tailings, and the new use that has been proposed for them. In this document, the authors also conclude the analysis and propose the creation of a new business in the mining value chain, so as to stimulate the sustainable development of this activity.

Keywords: Mining; Value chain; Tailings.

1 Membro da ABM, Engenheiro Químico, Doutor em Ciências, Resp. pelo Laboratório de Processos Metalúrgicos, Instituto de Pesquisas Tecnológicas do Estado de São Paulo, São Paulo - SP, Brasil.

2 Doutor em Engenharia de Produção, Gerente de Planejamento e Negócios do IPT - Instituto de Pesquisas Tecnológicas do Estado de São Paulo, São Paulo - SP, Brasil.

3 Engenheiro de Minas, Doutor em Ciências, Pesquisador, Instituto de Pesquisas Tecnológicas do Estado de São Paulo, São Paulo - SP, Brasil.

4 Engenheiro Químico, Pesquisador, Instituto de Pesquisas Tecnológicas do Estado de São Paulo, São Paulo - SP, Brasil.

5 Geólogo, Doutor em Geociências, Pesquisador, Instituto de Pesquisas Tecnológicas do Estado de São Paulo, São Paulo - SP, Brasil.. 


\section{INTRODUÇÃO}

Os dados desse projeto estruturados no documento intitulado "Rejeitos de Mineração: Um Olhar do Cenário Brasileiro - Parte I: Cadeia Produtiva" destacaram a importância econômica e social do setor mineral para o Brasil. De fato, para a manutenção do padrão atual de vida da sociedade, cada brasileiro consome cerca 5,4 toneladas de insumos de origem mineral, perfazendo um consumo total de 328 toneladas ao longo de uma vida de 70 anos. No entanto, para obtenção desses materiais são gerados 3.000 t de rejeitos por habitante.

Dados desse mesmo levantamento apontam que apenas 15 substâncias, das 70 processadas no país, geraram, em 15 anos, cerca de 4,86 bilhões de toneladas de rejeitos, sendo que as produções de ferro, ouro e fosfato representam $58 \%$ do total gerado.

É consenso que os minerais são recursos naturais finitos e que os teores dos minerais de interesse das reservas estão cada vez mais baixos. Uma abordagem que tem sido difundida neste setor é o reconhecimento dos rejeitos da mineração como minérios de baixo teor. Dentre os exemplos, destacam-se: ouro, zinco, carvão, ferro. Porém, para beneficiar e 'reconcentrar' o mineral útil contido no rejeito, as técnicas atuais deverão ser modificadas para que seja possível trabalhar com partículas finas e ultrafinas, especificamente a flotação.

Outro ponto fundamental para explorar o potencial ambiental e econômico dos rejeitos minerais é a redução do volume das barragens de rejeitos. Este tópico é tão importante que há uma lei, em vigor desde 2010, que estabelece a Política Nacional de Segurança de Barragens (Lei $n^{\circ}$ 12.334). As barragens de rejeitos oferecem sérios riscos se não forem planejadas corretamente e, posteriormente, operadas e mantidas. Segundo o PNRS, mais de 60 barragens, somente em MG, apresentam problemas de estabilidade. $O$ aproveitamento dos rejeitos como um minério de baixo teor reduziria a quantidade de rejeito alocado nas barragens, amenizando as dificuldades de manutenção e estabilidade.

O reaproveitamento e a disposição de rejeitos minerais de uma forma eficiente, ambientalmente e socialmente responsável, são, também, oportunidades para o desenvolvimento da competitividade industrial.

Segundo a Confederação Nacional da Indústria [1], o conhecimento e a inovação são motores da economia, em projeção realizada para o período de 2013 a 2022. Além disso, investimentos cada vez maiores deverão ser realizados em pesquisa e desenvolvimento de tecnologias que minimizem os danos ao meio ambiente, além da necessidade dos processos produtivos se adaptarem a padrões internacionais de eficiência ecológica.

Nesse contexto, o presente trabalho procurou trazer uma reflexão sobre o cenário atual da cadeia produtiva da mineração, a questão da geração de resíduos e o nível de desenvolvimento tecnológico voltado para aproveitamento desses rejeitos. Os dados levantados geraram uma série de informações que foram estruturadas em duas partes. Esse documento apresenta a Parte II dos levantamentos, a qual aborda o estágio atual de desenvolvimento tecnológico com vistas ao aproveitamento de rejeitos de mineração e os novos usos que têm sido propostos para esses materiais, assim como identifica os desafios impostos nesse contexto. Ao final, os autores trazem o fechamento das descobertas proporcionadas pelo projeto desenvolvido (Partes I e II) de forma integrada, colocando uma provocação com vistas a estimular o desenvolvimento sustentável da mineração. 


\section{MATERIAIS E MÉTODOS}

O conteúdo apresentado nesse documento está baseado nos seguintes aspectos:

- tecnologias empregadas no beneficiamento mineral e a sua relação com a eficiência dos processos;

- tecnologias avaliadas e empregadas para o aproveitamento de rejeitos e seus usos.

Para a realização dos levantamentos foram consultadas diversas bases de dados secundárias (banco de artigos e base de patentes) e também levantados dados primários por meio de entrevistas com os profissionais que atuam na área e especialistas do setor.

O levantamento realizado visou identificar como o tema de rejeito da mineração tem sido abordado nos meios científico e empresarial, para identificar as inovações propostas e em qual escala de processamento têm sido avaliadas. Além disso, buscou-se verificar os novos usos sugeridos para os rejeitos das substâncias minerais processadas.

\section{RESULTADOS E DISCUSSÃO}

Raramente as espécies minerais se encontram puras na natureza. Após a lavra do minério é necessário separar o mineral de interesse dos demais minerais presentes no minério, denominados minerais de ganga. Essa etapa, que consiste no que se convencionou chamar tratamento de minérios ou beneficiamento mineral, tem como objetivo obter o valor máximo de um dado bem mineral presente em uma mistura de espécies minerais. Tratam-se de operações aplicadas aos bens minerais que visam modificar sua granulometria, a concentração relativa das espécies minerais presentes ou sua forma, sem alterações químicas [2].

Ao final do processo de beneficiamento, obtém-se um concentrado do mineral de interesse. Os rejeitos do processo são encaminhados para as barragens, que são estruturas geotécnicas localizadas em bacias ou vales, destinadas à retenção da mistura de resíduos sólidos e água resultante dos processos de beneficiamento de minérios.

\subsection{Tecnologias de beneficiamento mineral}

Não é possível falar de tecnologias de aproveitamento de rejeitos sem destacar as tecnologias aplicadas em beneficiamento mineral. Tais tecnologias são desenvolvidas com base nas diferenças de propriedades apresentadas pelas espécies minerais. A rota tecnológica de concentração mineral pode se dar por processamento a úmido ou a seco, e está diretamente relacionada com o teor contido e a granulometria de liberação do bem mineral de interesse. Tradicionalmente, os processos de concentração mais utilizados são conduzidos a úmido, isto é, com a adição de quantidade significativa de água para formar a polpa, ou ainda nos processos de lavagem.

A granulometria de liberação dos bens minerais, geralmente, é da ordem de $100 \mu \mathrm{m}$. No entanto, em decorrência do empobrecimento das jazidas verificado nas últimas décadas, têm-se lavrado e beneficiado minérios complexos finamente disseminados, cujo intervalo de liberação é da ordem de $100 \mu \mathrm{m}-10 \mu \mathrm{m}$. Com isso, novos equipamentos visando a micronização de materiais têm sido desenvolvidos, sendo que há tecnologia disponível para processar materiais com granulometria da ordem de $2 \mu \mathrm{m}$. 
As tecnologias empregadas atualmente para recuperar metais contidos em minérios complexos são clássicas, complementares e bem definidas. O Quadro 1 resume as tecnologias empregadas para recuperar metais em minérios em função das propriedades diferenciadoras dos minerais.

As separações físicas são e ainda serão as mais empregadas no processo de industrial de produção mineral, as quais são agrupadas em duas categorias: gravítica e magnética, e flotação [3].

Quadro 1. Tecnologias empregadas para recuperar metais em minérios em função das propriedades que os diferenciam.

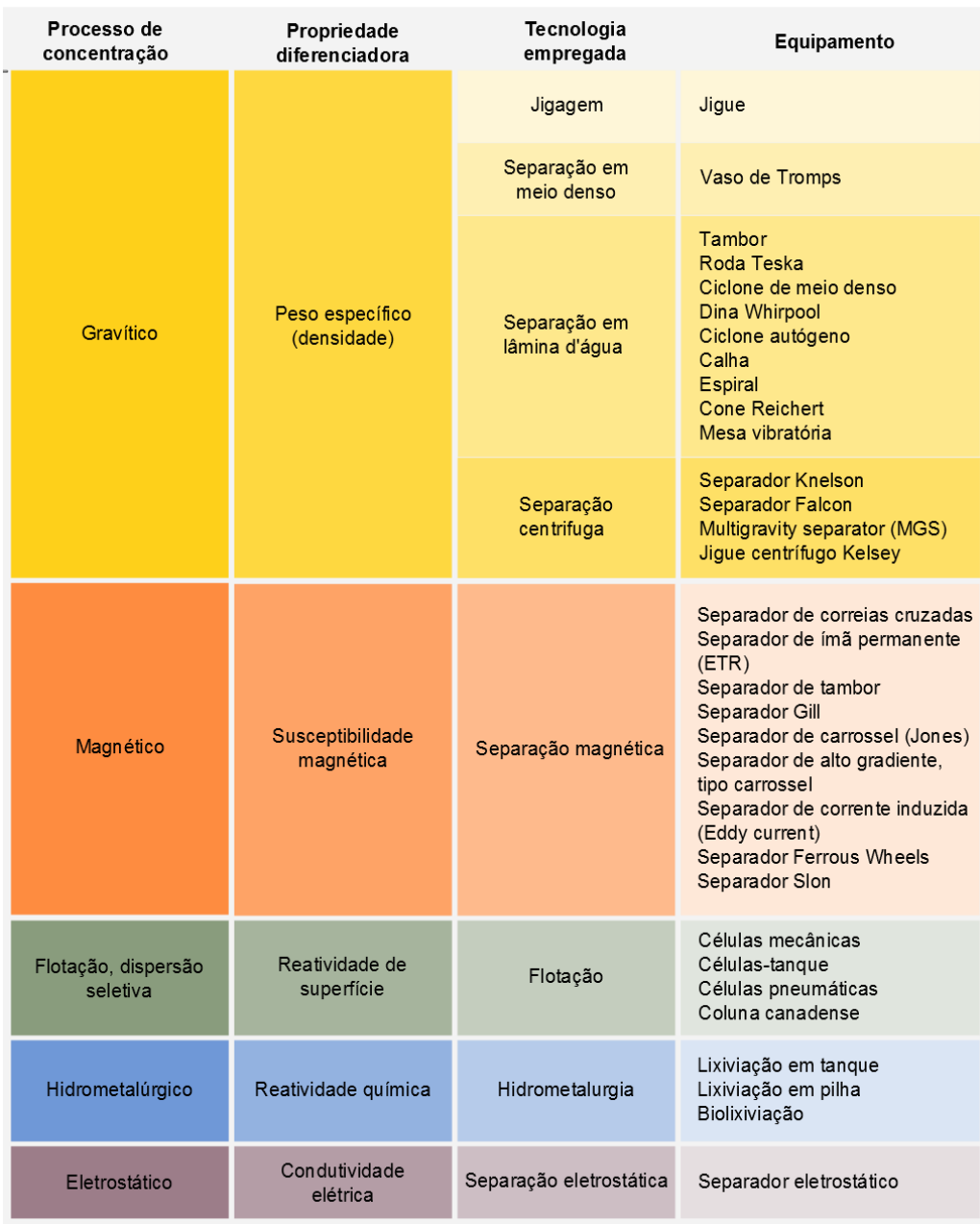

Fonte: Elaborado pelos autores com dados de Chaves e Chaves Filho [4] e Ciminelli [5].

As separações gravíticas e magnéticas são as tecnologias de separação mineral mais antigas e maduras. Essas tecnologias, além de serem aplicáveis a uma série de bens minerais, dispensam o uso de reagentes químicos, reduzindo o impacto ambiental negativo durante a disposição dos rejeitos. Em ambas as técnicas de separação, magnética ou gravítica, a seleção do equipamento a ser empregado é uma função do tamanho e das formas das partículas a serem separadas, do valor do bem mineral e da eficiência pretendida do processo.

Os equipamentos de separação gravítica mais modernos são o Jigue Centrífugo Kelsey e os separadores Knelson e Falcon. Dependendo da densidade do bem mineral, estes equipamentos são capazes de processar minérios com granulometria entre $50 \mu \mathrm{m}$ a $15 \mu \mathrm{m}[3]$. 
Os separadores magnéticos de alto gradiente (HGMS) foram introduzidos na década de 70 e têm estendido a aplicabilidade da separação magnética para recuperar materiais fracamente paramagnéticos.

Em relação à técnica de flotação, Negeri et al. [3] descrevem que, apesar de se tratar de uma disciplina estabelecida, muito difundida e, amplamente estudada mundialmente há mais de um século, essa técnica ainda não foi completamente entendida. Além disso, a eficiência da separação por flotação está sujeita à seletividade proporcionada pelos vários reagentes químicos usados, seguida de três fases de mistura hidrodinâmica e dependente da eficiência do equipamento de flotação e da sua configuração. Outra característica do processo de flotação é que esta técnica não é muito eficiente para minerais cuja granulometria de liberação seja muito fina.

No que diz respeito a tecnologias de processamento mineral, dois documentos valem ser destacados. Um deles, o roadmap de tecnologias de processamento mineral, construído em 2000 pela National Mining Association, em conjunto com o Departamento de Energia dos Estados Unidos, traz a visão da Indústria Mineral do Futuro daquele país. Outro documento, elaborado pelo Canada Mining Innovation Council em 2008, abordou os aspectos de inovação, pesquisa e desenvolvimento necessários nas áreas de processamento mineral e metalurgia extrativa para aquele país. A Figura 1 resume a análise desses documentos, destacando os desafios relacionados ao processamento de minérios complexos finamente disseminados e minérios marginais e as necessidades de pesquisa, desenvolvimento e inovação.

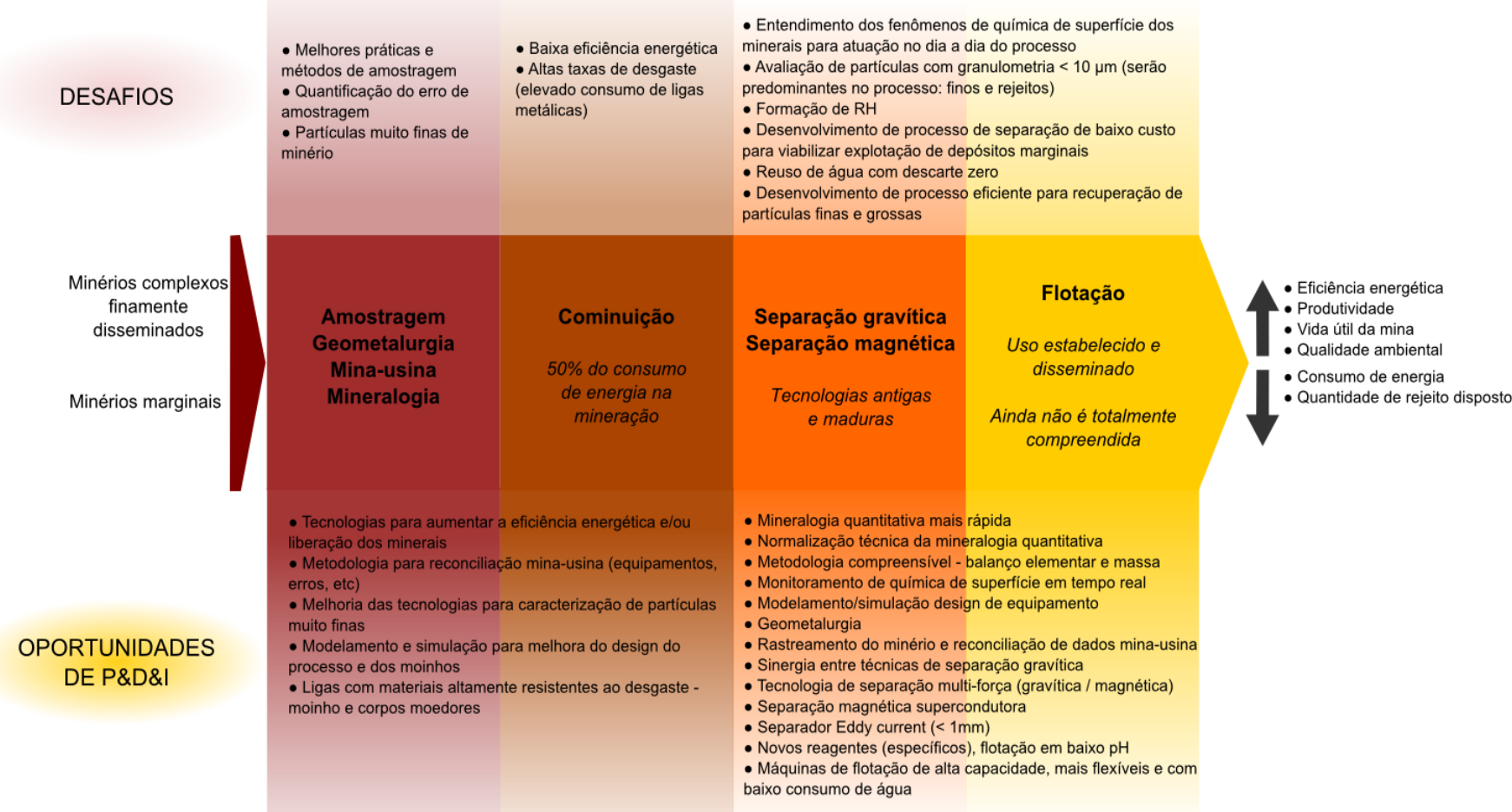

Figura 1. Desafios e oportunidades no processamento de minérios complexos finamente disseminados e minérios marginais. Fonte: Elaborado pelos autores.

Os desafios e as necessidades de P\&D\&I colocados na Figura 1 integram as várias etapas do processamento mineral, da mina aos produtos gerados, buscando aumento de eficiência, redução de consumo de energia, aumento da vida útil da mina e redução da quantidade de rejeitos a serem dispostos. Ressalta-se a 
necessidade apontada de desenvolvimento de tecnologias para separar minerais com granulometria finas da ordem de $20 \mu \mathrm{m}$ a $5 \mu \mathrm{m}$ e $<5 \mu \mathrm{m}$.

\subsection{Tecnologias Aplicadas para Aproveitamento de Rejeitos}

Idealmente os rejeitos não deveriam conter as espécies de interesse, mas verificase, porém, um alto porcentual desses minerais que não são recuperados nos processos de concentração e se reportam a esse fluxo. No caso do minério de ferro, o teor de ferro no rejeito pode variar de 10 a $60 \%$ [6]. Nas décadas passadas, estima-se que $1 / 3$ do fosfato, $1 / 6$ do cobre, $10 \%$ do minério de ferro lavrado nos EUA, $50 \%$ do estanho minerado na Bolívia e $20 \%$ do tungstênio lavrado no mundo, além de milhões de toneladas de outros minerais, foram perdidos nos finos, ou seja, na forma de rejeito [7]. Além do teor elevado, outra característica fundamental desse tipo material é sua pequena granulometria, constituído principalmente por partículas com tamanho da ordem de dezenas de micrometros.

Quanto ao tamanho, Wolff [6] afirma que a indústria mineral tradicionalmente designa os rejeitos da seguinte forma: rejeitos grossos ou granulares (maiores que $50 \mu \mathrm{m}$ ); rejeitos finos (entre 10 e $50 \mu \mathrm{m}$ ) e lamas ou rejeitos ultrafinos (partículas menores que $10 \mu \mathrm{m})$.

Wang et al. [8] em sua revisão crítica sobre o estado atual do tratamento de finos de barragens traz as proporções e características típicas de rejeitos gerados durante 0 processamento de alguns minerais no mundo, resumidos na Tabela 1.

Tabela 1. Proporções e características mundiais de rejeitos estocados em barragens.

\begin{tabular}{|c|c|c|c|}
\hline $\begin{array}{l}\text { Mineral } \\
\text { processado }\end{array}$ & $\begin{array}{l}\text { Proporção rejeito/tonelada } \\
\text { processada de minério } \\
\text { (kg/tonelada) }\end{array}$ & \% sólidos (w/w) & Distribuição granulométrica \\
\hline Carvão & $75-120$ & $5-55$ & $>75 \mu \mathrm{m}(19 \%)$ até $<4 \mu \mathrm{m}(66 \%)$ \\
\hline Bauxita & $\begin{array}{l}1-1,5 \\
\text { Lama vermelha/alumina produzida }\end{array}$ & $20-80$ & $\begin{array}{l}2-100 \mu \mathrm{m} \\
<5 \mu \mathrm{m}(50 \%)\end{array}$ \\
\hline Cobre & $128-196$ & & $>75 \mu \mathrm{m}(66 \%)$ até < $1 \mu \mathrm{m}(34 \%)$ \\
\hline Fosfato & 375 & $5-45$ & $>75 \mu \mathrm{m}(66 \%)$ até $<37 \mu \mathrm{m}(57 \%)$ \\
\hline Ferro & $450-500$ & $30-50$ & $<10 \mu \mathrm{m}$ \\
\hline
\end{tabular}

Assim como o teor do bem mineral de interesse contido no rejeito, as características físico-químicas apresentadas por eles tem impacto direto na definição da rota tecnológica a ser aplicada para a sua valoração.

Por se tratarem de materiais descartados pelos processos de beneficiamento mineral, os rejeitos apresentam alterações devido aos processos de cominuição e concentração, afetando as suas propriedades superficiais e morfológicas. Esse é um fator determinante quando o que se busca são alternativas voltadas para a sua recuperação. Isso porque o beneficiamento de materiais ultrafinos necessita de processos diferentes dos utilizados comumente nas plantas de beneficiamento mineral, uma vez que estas foram dimensionadas para operar em faixas granulométricas mais grossas, entre $50 \mu \mathrm{m}$ e $100 \mu \mathrm{m}$ (Figura 2).

Ainda assim, a tentativa de valoração de rejeitos gerados pela mineração tem sido amplamente avaliada no Brasil e no mundo. Há uma abundância de artigos técnicocientíficos abordando o tema, tanto na linha de recuperação do bem mineral de interesse contido no rejeito, quanto na sua aplicação nos mais variados segmentos industriais, o que será discutido mais adiante nesse documento.

Com este olhar, nesse trabalho foi realizado um levantamento visando identificar como o tema rejeitos da mineração tem sido abordado no meio científico, quais inovações têm sido propostas no se refere a tecnologias para recuperação de bens 
minerais de interesse, bem como em quais escalas de processamento têm sido avaliadas. Buscou-se, também, verificar quais novos usos estão sendo propostos e a partir de rejeitos de quais substâncias minerais processadas. Uma análise crítica das patentes solicitadas no tema e dos artigos técnicos internacionais publicados em diferentes bases de dados foi realizada.

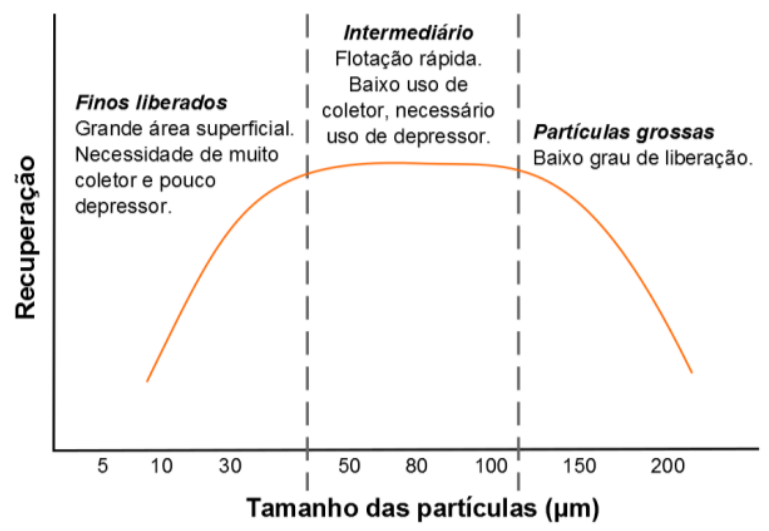

Figura 2. Recuperação na flotação em função do tamanho de partícula. Fonte: adaptado de Fuerstenau [7].

\subsubsection{Análise de patentes sobre o tema rejeitos da mineração}

A busca por patentes sobre o tema rejeitos da mineração foi realizada no mês de novembro de 2016 na plataforma Orbit, utilizando como critério de busca as palavras- chave: mineral process, mineral processing, mineral dressing, ore process, ore processing, ore dressing, cada uma associada às palavras tailings ou waste, por meio do seguinte operador lógico: (Mineral or Ore) And (Process+or Dressing) and (Tailings or Waste). A busca foi realizada no título, assunto e resumos das patentes, considerando somente as patentes ativas ("alive" = granted ou pending). O resultado da busca retornou 11.028 patentes que atenderam aos critérios selecionados.

O objeto dessas patentes enquadra-se em dois domínios tecnológicos principais: "Materiais e metalurgia" e "Engenharia química".

Mais da metade das patentes foram requeridas a partir de 2011 (Figura 4), denotando o crescente interesse do mercado no assunto nos últimos anos. Também, cerca de metade das patentes foram depositadas primeiro na China.

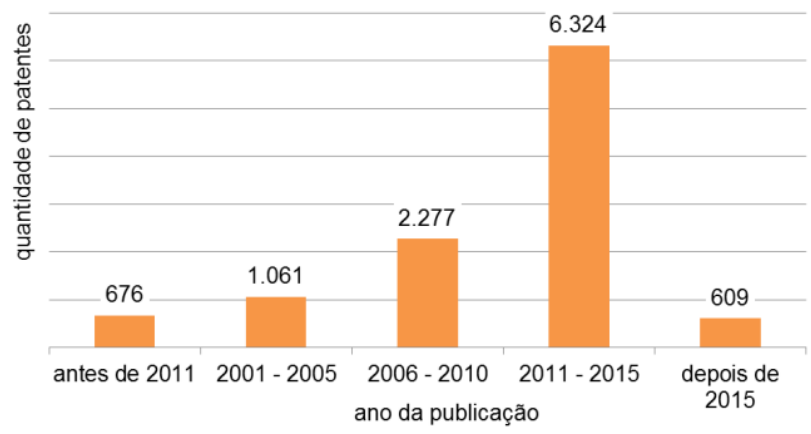

Figura 4. Ano do primeiro pedido de depósito (1st year application) das patentes localizadas.

Considerando-se a classificação dessas 11.028 patentes de acordo com o CPC Cooperative Patent Classification, verifica-se que $36 \%$ estão classificadas na seção $\mathrm{Y}$, ou seja, referem-se a novos desenvolvimentos tecnológicos (ou tecnologias) interdisciplinares, sendo $34 \%$ classificados com Y02 - Tecnologias para mitigação ou adaptação às mudanças do clima, dos quais $18 \%$ na produção ou processamento de bens e $16 \%$ relacionados ao tratamento de água ou gerenciamento de resíduos, conforme a Figura 5. 
A partir dessa classificação, observa-se que parte significativa das patentes no tema rejeitos de mineração considera uma solução interdisciplinar para o problema. Quanto às tecnologias envolvidas, verifica-se a utilização da separação magnética, além da utilização de técnicas hidrometalúrgicas, corroborando os levantamentos que foram obtidos do estado da técnica e nos artigos científicos e que são apresentados no item 3.2.1, desse documento.

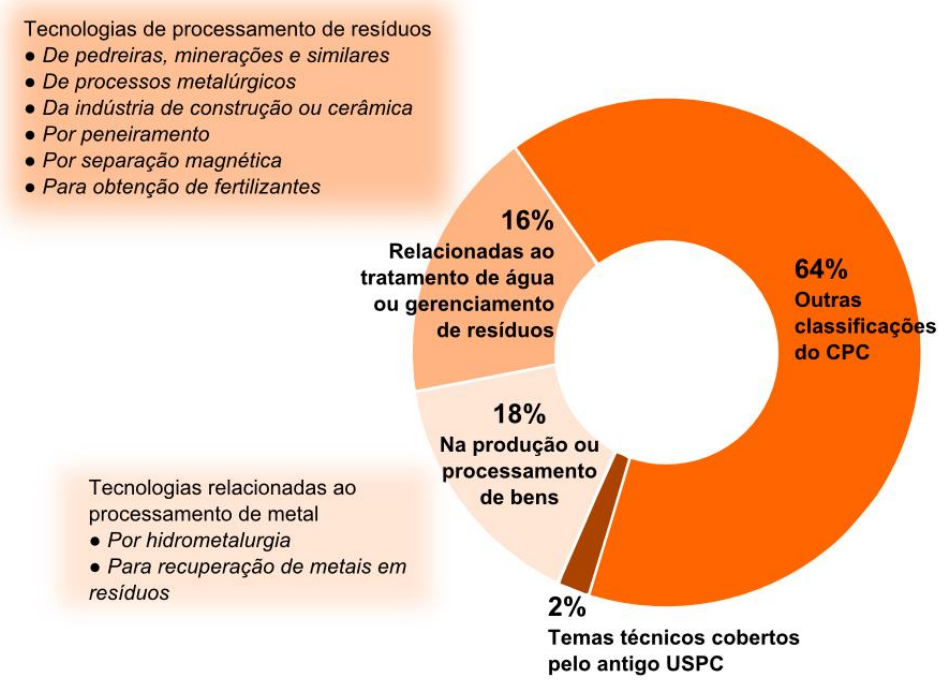

Figura 5. Classificação CPC das patentes em rejeito da mineração.

\subsubsection{Análise das publicações técnico-científicas sobre o tema rejeitos da mineração}

Com o objetivo de delinear o estado da arte das tecnologias utilizadas em beneficiamento mineral, seja para processamento do minério ou dos rejeitos, foi realizada uma busca de artigos científicos na base de dados Scopus. Inicialmente, a busca foi realizada a partir de quatro assuntos utilizados como palavras-chave ("mineral tailings recovery", "fine particles recovery", "mineral processing" e "mineral dressing"), tendo sido localizados mais de 9.000 artigos. Essa busca foi refinada (Quadro 2), limitada aos últimos trinta anos e restringindo-se a quantidade de periódicos e as substâncias minerais, buscando abranger aquelas em que o Brasil é um produtor destacado e aquelas em que o país é deficitário.

Quadro 2. Critérios de busca por artigos científicos - últimos 30 anos.

\begin{tabular}{|c|c|cc|}
\hline Assunto & Periódico & \multicolumn{2}{|c|}{ Substância mineral } \\
\hline Mineral tailings recovery & Revista Escola de Minas - REM & Bauxite & Kaolin \\
Fine particles recovery & Minerals Engineering & Coal & Limestone \\
Mineral processing & International Journal of Mineral Processing & Copper & Niobium \\
Mineral dressing & Mineral Processing and Extractive Metallurgy & Gold & Phosphate \\
& The Metals Society - TMS Annals & Iron & \\
& Advanced Materials Research & & \\
& &
\end{tabular}

A nova busca resultou em 507 artigos (485, quando excluídas as repetições, ou seja, aqueles artigos que foram recuperados para mais de uma substância mineral). Dessas publicações, 280 (58 \%) são dos últimos dez anos.

Os artigos publicados (Figura 6) concentram-se nos períodos 2009-2013 (Fe, Au e Cu) e 2001-2003 (carvão). Considerando que os investimentos em pesquisa são também um reflexo da economia, pode-se explicar essa concentração de artigos com base na tendência de crescimento da mineração no mercado mundial, que se iniciou em 2000 e prosseguiu até 2011, alavancado pelos maciços investimentos em infraestrutura realizados pela China no período. Após a consolidação da alta da 
mineração no mercado, o setor passou a investir mais em pesquisas na área, principalmente naquelas consideradas menos atrativas, como os rejeitos. Esse processo explicaria o grande número de publicações de resultados publicados em periódicos no período entre 2009-2013. Embora o período da alta da mineração tenha terminado em 2011, é necessário considerar que os resultados das pesquisas demandam um tempo até gerarem publicação, pois deve-se somar após a finalização dos projetos um prazo adicional que é o tempo inerente ao trâmite de publicação de artigos científicos em periódico internacional. Devido ao declínio da mineração no mercado mundial, que vem sendo observado desde meados de 2011, espera-se uma tendência de redução na quantidade de artigos publicados no tema considerado nos próximos anos.

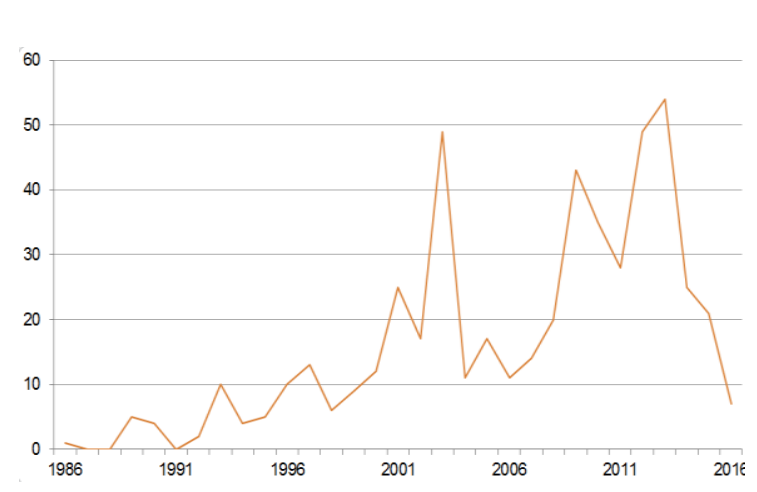

Figura 6. Quantidade de artigos localizados por ano no período $1986-2016$.

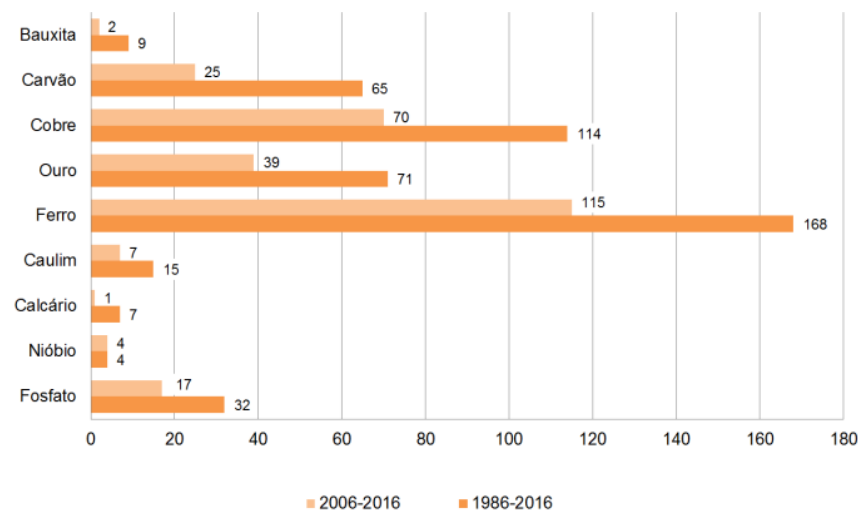

Figura 7. Quantidade de artigos localizados, por substância mineral, nos últimos trinta e dez anos.

As principais substâncias minerais consideradas pelos artigos localizados foram ferro $(34,6 \%)$, cobre $(23,5 \%)$, ouro $(14,6 \%)$ e carvão $(13,4 \%)$, conforme apresentado na Figura 7. A mesma figura também mostra que, quando se consideram as publicações referentes aos últimos 10 anos, as mesmas substâncias são objeto de $88,9 \%$ das publicações. Pode-se associar a frequência das publicações aos principais usos dessas substâncias: infraestrutura (ferro e carvão na siderurgia, cobre para cabos elétricos) e energia (carvão), enquanto o ouro é um metal precioso, usado como ativo na economia.

Dos 485 artigos localizados pela busca, foram selecionados aleatoriamente 34 para leitura, respeitando a proporção aproximada entre os artigos relacionados a cada um dos bens minerais na busca principal. A partir dos artigos selecionados para leitura, foi elaborada uma análise que buscou identificar quais as tecnologias e equipamentos aplicados ao processamento do rejeito foi considerado pelo artigo, em qual escala foi realizada a pesquisa, e se essa resultou no reaproveitamento do rejeito ou em um novo produto, identificando também, nesse caso, qual seu mercado.

Dos artigos avaliados, a China se destaca como o país que mais publica artigos científicos relativos ao tema de rejeitos da mineração, com $32 \%$ das publicações analisadas. O Canadá ocupa o segundo lugar com $17 \%$ e o Brasil está na terceira posição, com $12 \%$ dos trabalhos publicados (mas cabe a ressalva que uma das publicações utilizadas como fonte dos artigos é nacional). Os países Austrália, França, Índia e Rússia contribuíram, cada um, com $6 \%$ dos artigos publicados, enquanto que a África do Sul, Chile, Grécia, Japão e Reino Unido, publicaram $3 \%$ dos artigos, cada um. Ressalta-se que a grande maioria dos artigos chineses analisados fazia referência a modificações em separadores magnéticos de alta intensidade. 
Em relação ao tipo de rejeito gerado, ou seja, fonte do processamento de qual bem mineral, observa-se na Figura 8 que a maior parte $(31 \%)$ das publicações trata do rejeito de minério de ferro, seguido do rejeito de cobre (13\%). Os rejeitos de bauxita, carvão, níquel e ouro aparecem com $8 \%$ dos artigos cada, seguidos por zinco e caulim (5\%) Já os rejeitos elementos de terras raras (ETR), fosfato, manganês, pedra calcária, quartzo e tungstênio foram alvo de $3 \%$ dos estudos, cada um. Nessa figura, a totalização dos artigos é superior à quantidade de artigos selecionados, pois alguns deles tratam de mais de uma substância mineral.

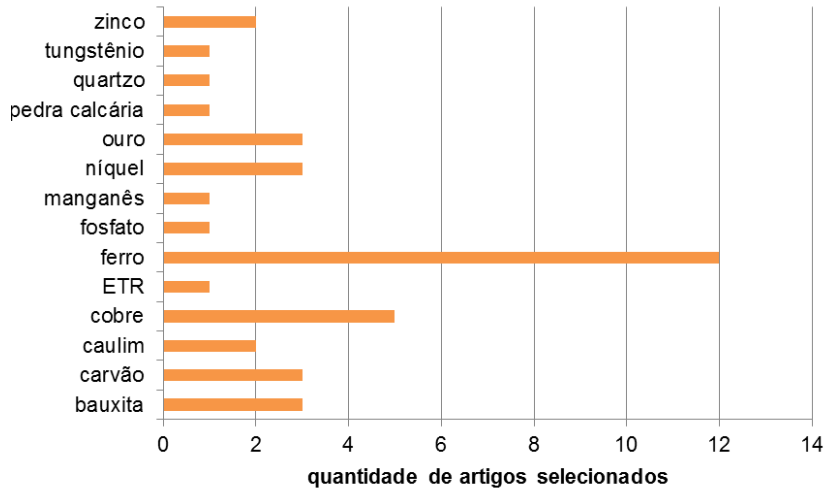

Figura 8. Fonte de rejeito, por bem mineral, tratada nos artigos técnico-científicos analisados.

A Figura 9 sintetiza informações relevantes da busca de artigos, destacando o emprego de tecnologias tradicionais de tratamento de minérios visando obter concentrados a partir de rejeitos variados. Quanto às tecnologias abordadas nos artigos, há um forte predomínio $(41,5 \%)$ do uso de separações gravíticas ou magnéticas (respectivamente $22,6 \%$ e 18,9\%). A busca não encontrou nenhuma inovação radical de tecnologias de processamento mineral.

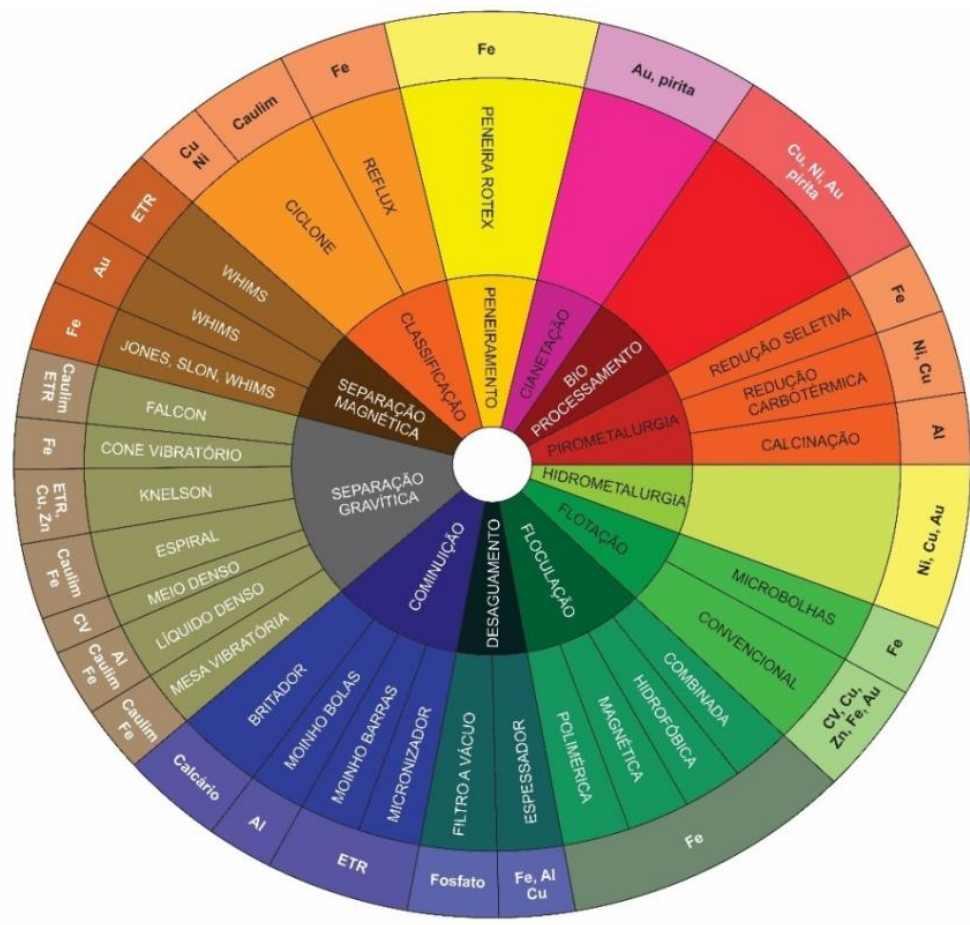

Figura 9. Síntese das tecnologias de aplicadas ao tratamento de rejeitos nos artigos analisados. Destaque para o emprego de técnicas consagradas do beneficiamento mineral. Fonte: elaborado com informações de [11] - [43]. 
Dentre os novos usos dados aos rejeitos, após processamento, destacam-se aqueles que têm como mercado-alvo a indústria de construção civil e a obtenção de concentrados para as indústrias metalúrgica e siderúrgica. A Figura 10 resume 0 nível de desenvolvimento tecnológico apresentado nos artigos, assim como os tipos de rejeitos abordados e os novos usos propostos.

Os artigos selecionados foram classificados em teóricos (8 artigos de revisão), prospecção de mercado (1 artigo) ou trabalhos experimentais (25 artigos). Desses últimos, $64 \%$ foram desenvolvidos em escala de bancada, $20 \%$ em escala industrial e $4 \%$ em modelo físico. Utilizando-se a denominação dos TRL (Technology Readiness Levels) é possível afirmar que a grande maioria dos artigos apresentam resultados de desenvolvimento de tecnologia ( $T R L 1$ a 4) e no estágio inicial de desenvolvimento exploratório (TRL 5).

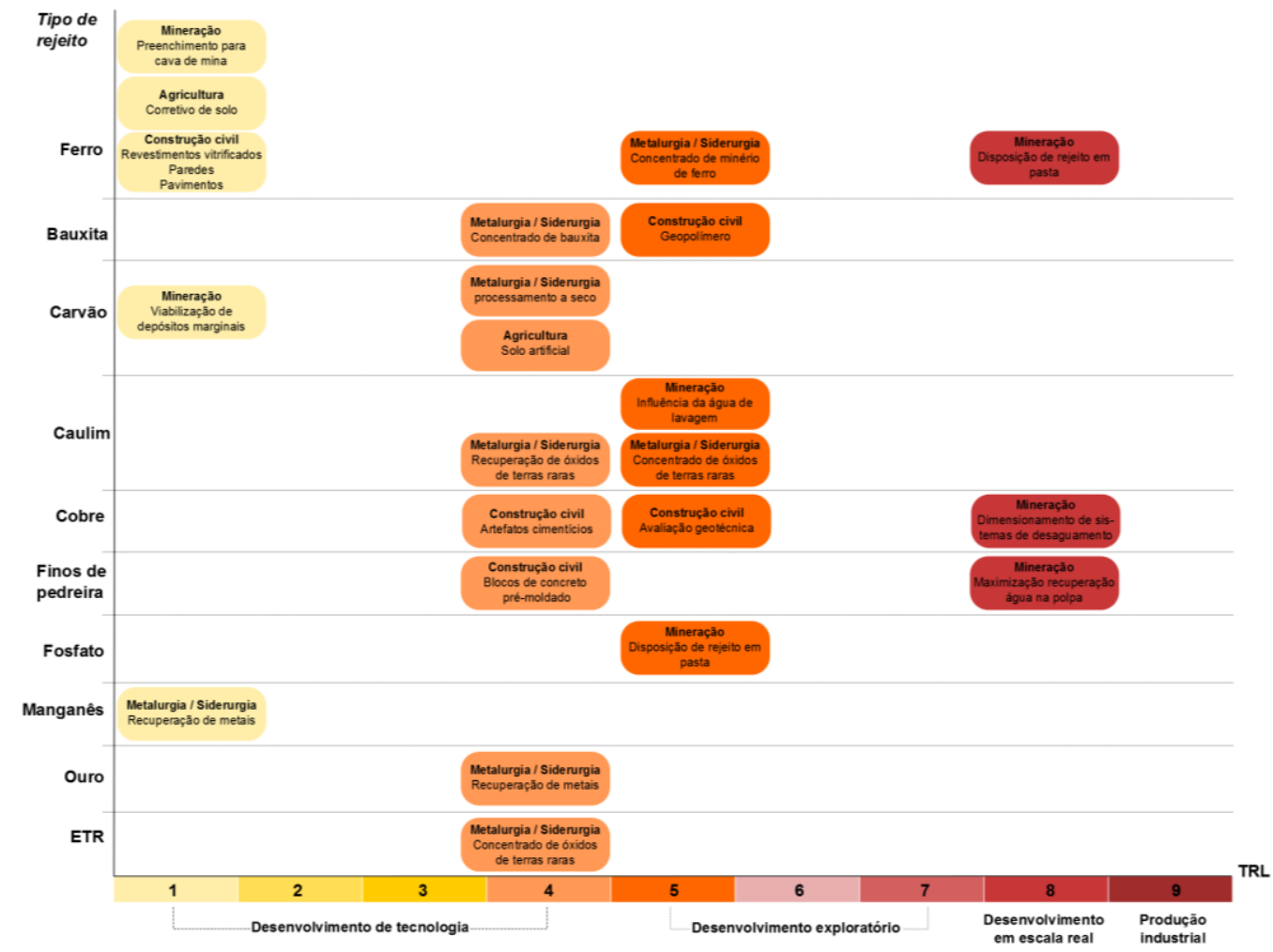

Figura 10. Nível de desenvolvimento tecnológico apresentado nos artigos, tipos de rejeitos abordados e novos usos propostos.

\section{CONCLUSÃO}

Os minerais são recursos naturais e são finitos. O empobrecimento das jazidas tem levado ao processamento de minérios cuja granulometria passou de cerca de $100 \mu \mathrm{m}$ para algo da ordem de $100 \mu \mathrm{m}$ a $10 \mu \mathrm{m}$. No entanto, as plantas de beneficiamento mineral foram dimensionadas para operar em faixas granulométricas entre $50 \mu \mathrm{m}$ e $100 \mu \mathrm{m}$. Esse fato, aliado ao teor do bem mineral contido e outros fatores relativos à eficiência do processo, fazem com que os rejeitos tenham alto percentual de material de interesse que não foram recuperados e são descartados em pilhas ou barragens de rejeitos. As discussões colocadas em documentos redigidos por técnicos de países com tradição mineral apontam como grande desafio a melhoria da eficiência dos processos, sendo que a necessidade de desenvolvimento de tecnologias para processar materiais finamente disseminados é discutida desde a década de 2000. 
Os dados levantados nesse trabalho indicam que muitos estudos técnico-científicos têm sido realizados mundialmente no tema rejeitos, sendo que o rejeito de minério de ferro é o mais investigado.

As investigações que visavam concentrar minerais contidos nos vários tipos de rejeito fizeram uso de técnicas convencionais de beneficiamento, não indicando nenhuma inovação radical de processamento mineral. A China se desponta nesses estudos, principalmente no desenvolvimento de equipamentos de separação magnética de alta intensidade. Outro fato importante constatado se refere ao nível de desenvolvimento tecnológico das rotas de aproveitamento propostas nesses estudos, na sua grande maioria em nível de desenvolvimento da tecnologia e exploratório, ou seja, teórico ou nível de bancada.

Ainda que o país seja detentor de um arcabouço legal robusto e, que a tragédia do acidente ocorrido com a barragem de rejeitos da Samarco tenha fomentado a discussão sobre a problemática da disposição de rejeitos da mineração, o problema está muito longe de ser equacionado.

A análise da situação e das ações dos atores que compõem a cadeia mineral permite inferir uma provocação quanto a inexistência nessa cadeia de um elo voltado ao aproveitamento de rejeitos. Como pode ser observado na Figura 11, 0 aproveitamento de rejeitos não figura de maneira explícita na cadeia mineral, como um negócio, nem para as empresas de mineração e nem para um terceiro ator nesse processo.

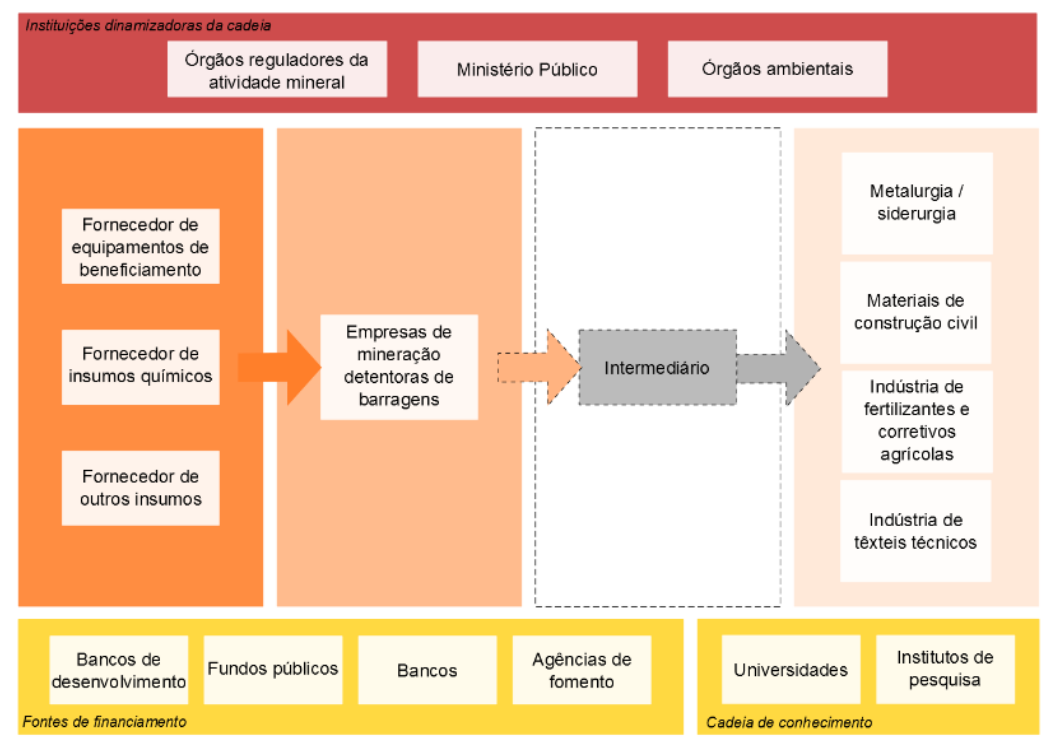

Figura 11. Cadeia produtiva do setor mineral. Destaca-se a inexistência do setor de aproveitamento de rejeitos enquanto um negócio, representado pela caixa cinza denominada intermediário na Figura.

Nesse caso, na visão dos autores desse trabalho, a caixa denominada "intermediário" na Figura 11 pode ser desenvolvida enquanto um novo negócio, seja para o seu gerador, para os usuários das matérias-primas minerais ou mesmo para um novo elo dessa cadeia. Para isso, entende-se que a abordagem de Economia Circular é extremamente aderente a esse contexto. A Economia Circular se baseia na ideia de desenvolvimento como um ciclo positivo contínuo que preserva e aprimora o capital natural, otimiza a produção de recursos e reduz riscos sistêmicos administrando estoques finitos e fluxos renováveis. Esse conceito abrange a necessidade de transformar os resíduos em insumos para a produção de novos 
produtos. Na Figura 12, colocam-se as oportunidades e desafios para a construção de tal modelo.

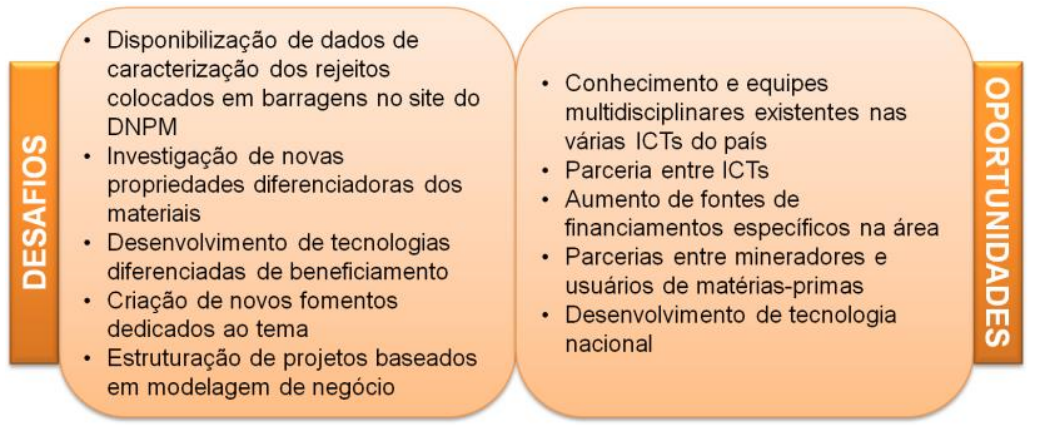

Figura 12. Oportunidades e desafios do novo modelo para cadeia de mineração.

\section{Agradecimentos}

Os autores agradecem ao Instituto de Pesquisas Tecnológicas do Estado de São Paulo, à Fundação de Apoio do Instituto de Pesquisas Tecnológicas do Estado de São Paulo e a Secretaria de Desenvolvimento Econômico, Ciência, Tecnologia e Inovação pelo financiamento do projeto "Mapeamento da produção de rejeitos das empresas de mineração e da maturidade das tecnologias aplicadas para sua valoração", que gerou os resultados apresentados nesse artigo.

\section{REFERÊNCIAS}

1 CNI. Mapa estratégico da indústria 2013-2022. Confederação Nacional da Indústria. 2013. [acesso em: 31 de maio 2017]. Disponível em: http://www.abit.org.br/adm/Arquivo/Servico/114357.pdf

2 Luz, AB; Lins, FAF. in: Luz, AB; Sampaio, JA; França, CA. Tratamento de minérios. Rio de Janeiro: CETEM/MCT, 2010. cap1. p.3-18, 2010.

3 Negeri T et al. Innovation, Research and development needs in mineral processing and extrative metallurgy. Canada Mining Innovation Council. 2008. [acesso em: 31 de maio 2017]. Disponível em:

https://connect.innovateuk.org/documents/2865354/7780642/Innovation,\%20Research+a nd+Development+Needs+in+Mineral+Processing+and+Extractive+Metallurgy/71 b890a7b9be-4f17-93a3-23ed728

4 Chaves AP; Chaves Filho RC. Teoria e prática de tratamentos de minérios. Separação densitária. Vol.6. São Paulo: Oficina de textos, 2013.

5 Ciminelli VST. Hidrometalurgia. Cap 4. CETEM. [acesso em: 31 de maio 2017].

Disponível em:

http://mineralis.cetem.gov.br/bitstream/handle/cetem/1288/Tend\%C3\%AAnciasParte2.4.p $\mathrm{df}$ ? sequence $=1$

6 Wolff AP. Caracterização de rejeitos de minério de ferro de minas da vale. Ouro Preto, 2009. 90p. Dissertação de mestrado - Universidade Federal de Ouro Preto, 2009.

7 Fuerstenau D.W. Flotation science and engineering: advances and challenges. 1988. In: Fossberg, K.S.E. (Ed.), XVI International Mineral Processing Congress, pp. 63-80, 1998.

8 Wang C. et al. Current state of fine mineral tailings treatment: A critical review on theory and practice. Minerals Engineering, 58, p.113-131, 2014.

9 Marques MS. Salitre Project - dewatered phosphate rocks tailings as alternatives to conventional tailings disposal, Vale Fertilizantes Minas Gerais State, Brazil. 2011. [acesso em: 31 de maio 2017]. Disponível em:

http://www.golder.com/en/modules.php?name=Publication\&op=showlivepdf\&sp_id=238\&l ang_id=3

10 Silva $A C$ et al. Mineral paste production from phosphate rock tailings. Mining Mineração.

R. Esc. Minas, Ouro Preto, 68, p. 103-108, 2015. 
11 Amariei D. et al. The use of a reflux classifier for iron ores: Assessment of fine particles recovery at pilot scale. Mineral Engineering. v. 62, p. 66-73, 2014.

12 Bazin C. et al. Size recovery curves of minerals in industrial spiral for processing iron oxide ores. Minerals Engineering. v. 65, p. 115-123, 2014.

13 Bulaev AG. et al. Bioprocessing of mining and metallurgical wastes containing non-ferrous and precious metals. Advanced Materials Research. v. 825, p. 301-304, 2013.

14 Chan BKC.; Bouzalakos S.; Dudeney AWL. Cemented products containing waste from mineral processing and bioleaching. Minerals Engineering. v. 22, p. 1326-1333, 2009.

15 Chen L.; Ren N; Xiong D. Experimental study on performance of a continuous centrifugal concentrator in reconcentrating fine hematite. International Journal of Mineral Processing. v. 87, p. 9-16, 2008.

16 Dehaine Q.; Filippov LO. Rare earth ( $\mathrm{La}, \mathrm{Ce}, \mathrm{Nd})$ and rare metals $(\mathrm{Sm}, \mathrm{Nb}, \mathrm{W})$ as byproduct of kaolin production, Cornwall: Part1: Selection and characterization of the valuable stream. Minerals Engineering. v. 76, p. 141-153, 2015.

17 Dehaine Q.; Filippov LO. Modelling heavy and gangue mineral size recovery curves using the spiral concentration of heavy minerals from kaolin residues. Powder Technology. $v$. 292, p. 331-341, 2016.

18 Donskoi E. et al. Iron ore textural information is the key for prediction of downstream process performance. Minerals Engineering. v. 86, p. 10-23, 2016.

19 Firpo BA.; Filho JRA.; Schneider IAH. A brief procedure to fabricate soils from coal mine wastes based on mineral processing, agricultural, and environmental concepts. Minerals Engineering. v.76 , p. 81-86, 2015.

20 GALETAKIS, M.; RAKA, S. Utilization of limestone dust for artificial stone production: an experimental approach. Minerals Engineering. v. 17, p. 355-357, 2004.

21 Gálvez ED. et al. Optimization of dewatering systems for mineral processing. Minerals Engineering. v. 63, p. 110-117, 2014.

22 Glosh S. et al. A greener approach for resource recycling: Manganese bioleaching. Chemosphere. v. 154, p. 628-639, 2016.

23 Izoitko VM; Shumskaya YN. Old tailings dumps of concentrating plants as a source of raw materials. Developments in mineral processing, 13, 2000.

24 Jaiswal S.; Tripathy SK.; Banerjee PK. An overview of reverse flotation process for coal. International Journal of Mineral Processing. v.134, p. 97-110, 2015.

25 Jordens $A$. et al. Processing a rare earth mineral deposit using gravity and magnetic separation. Minerals Engineering. v. 62, p. 9-18, 2014.

26 Kahn H.; Tassinari MML.; Ratti G. Characterization of bauxite fines aiming to minimize their iron content. Minerals Engineering. v. 16, p. 1313-1315, 2003.

27 Kökkiliç O.; Langlois R.; Waters KE. A design of experiments investigation into dry separation using a Knelson Concentrator. Minerals Engineering. v.72 , p. 73-86, 2015.

$28 \mathrm{Li} \mathrm{F}$. et al. Development and utilization of the micro-fine weakly magnetic iron ore. Advanced Materials Research. v. 734-737, p. 925-928, 2013.

$29 \mathrm{Liu}$ J. et al. A research on tailings reprocessing from Da-hongshan. Advanced Materials Research. v. 813, p. 221-224, 2013.

30 Liu S. et al. A combined beneficiation process to recover iron minerals from a finely disseminated low-grade iron ore. Advanced Materials Research. v. 634-638, p. 32733276, 2013.

31 Makinde OA.; Ramatsetse BI.; Mpofu K. Review of vibrating screen development trends: Linking the past and the future in mining machinery industries. International Journal of Mineral Processing. v. 145, p. 17-22, 2015.

32 Oshitani J. et al. Dry beneficiation of fine coal using density-segregation in a gas-solid fluidized bed. Advanced Powder Technology. v. 27, p. 1689-1693, 2016.

33 Peek E.; Barnes A.; Tuzum A. Nickeliferous pyrrhotite - "Waste or resource?" Minerals Engineering. v. 24, p. 625-637, 2011.

34 Ribeiro JP.; Ribeiro CHT. The NoBLOCK technology - A major breakthrough in wet high intensity magnetic separation (WHIMS). REM: Revista Escola Minas. v. 68, p. 361-366, 2015. 
35 Silva AC. et al. Mineral paste production from phosphate rock tailings. REM: Revista Escola de Minas. v. 68, n. 1, p. 103-108, 2015.

36 Xinhui D. et al. Research progress on resource utilization of iron tailings and its development trend in China. Applied Mechanics and Materials. v. 320, p. 780-784, 2013.

37 Yalcin T.; Kaukolin E.; Byers A. Axial inlet cyclone for mineral processing applications. Mineral Engineering. v. 16, p. 1375-1381, 2003.

38 Ye J; Zhang W. Shi D. Effect of elevated temperature on the properties of geopolymer synthesized from calcined ore-dressing tailing of bauxite and ground-granulated blast furnace slag. Contruction and building materials, 69, p. 41-48, 2014.

39 Yin G. et al. Stability analysis of a copper tailings dam via laboratory model tests: A Chinese case study. Minerals Engineering. v. 24, p. 122-130, 2011.

40 Zammit CM. et al. The future of biotechnology for gold exploration and processing. Minerals Engineering. v. 32, p. 45-53, 2012.

41 Zhang $\mathrm{H}$. Concentration on limonitic iron ore by multi-grade magnetic roasting-low intensity magnetic separation. Advanced Materials Research. v. 933, p. 125-131, 2014.

42 Zhou X. et al. Study on the vibration lamella thickener and its application in mineral processing plant. Applied Mechanics and Materials. v. 215-216, p. 333-341, 2012.

43 Zhou X. et al. Paste thickening iron tailings with vibrating lamella thickener. Advanced Materials Research. v. 690-693, p. 3570-3575, 2013. 